\title{
Wnt pathway-related three-mRNA clinical outcome signature in bladder urothelial carcinoma: computational biology and experimental analyses
}

Siqing Sun ${ }^{\dagger}$, Yutao Wang ${ }^{\dagger}$, Jianfeng Wang and Jianbin Bi ${ }^{*}$ (I)

\begin{abstract}
Background: The Wnt signaling pathway is core to the growth of bladder tumors. Epithelial-to-mesenchymal transition (EMT) is significant for bladder tumor metastasis. Nevertheless, the relationship between the Wnt signaling pathway, outcomes of bladder cancer (BLCA), and the specific mechanisms driving immune infiltration have not been studied.

Methods: We obtained Wnt pathway-related gene mRNA and clinicopathological data from the Cancer Genome Atlas (TCGA). We obtained 34 genes that were greatly correlated with outcome using univariate Cox regression analysis and conducted a completely randomized data t-test to perform clinical staging. According to the single-sample gene set enrichment analysis (ssGSEA), the weighted correlation network analysis (WGCNA) was applied to identify relevant biological functions. Various subtypes were identified using consensus cluster analysis. Univariate Cox regression and least absolute shrinkage sum selection operator-Cox regression algorithm analysis were conducted on TCGA and Gene Expression Omnibus data to identify risk characteristics. The Kaplan-Meier method and receiver running feature curves were adopted to calculate overall survival. Single-sample gene set enrichment analysis (ssGSEA) was adopted for the assessment of the degree of immune infiltration. Then, we demonstrated the relationship between PPP2CB and EMT function in two cell lines.
\end{abstract}

Results: Thirty-four Wnt signaling pathway-related genes were risk factors for BLCA outcome, and their expression levels differed by clinical stage. The co-expression of WGCNA showed the relationship between the Wnt signaling pathway and biological functions and was closely associated with EMT. We divided BLCA patients into two subtypes using consensus clustering. Survival curves and clinical analysis showed that the Wnt pathway enriched group had worse outcomes. The Wnt signature showed the significance of the outcome for MAPK10, PPP2CB, and RAC3. Based on these genes, the degree of immune infiltration was evaluated. Cell function experiments suggested that PPP2CB drives the proliferation and migration of BLCA cells.

Conclusion: We found that Wnt signaling pathway-related genes can be used as prognostic risk factors for BLCA, and the Wnt signaling pathway is a cancer-promoting signaling pathway associated with EMT. We identified three critical genes: MAPK10, RAC3, and PPP2CB. The genes in these three Wnt signaling pathways are associated with tumor cell

\footnotetext{
*Correspondence: jianbinbi@cmu.edu.cn

†'Siqing Sun and Yutao Wang contributed equally to this article Department of Urology, China Medical University, The First Hospital

of China Medical University, Shenyang 110001, Liaoning, China
} permits use, sharing, adaptation, distribution and reproduction in any medium or format, as long as you give appropriate credit to the original author(s) and the source, provide a link to the Creative Commons licence, and indicate if changes were made. The images or other third party material in this article are included in the article's Creative Commons licence, unless indicated otherwise in a credit line to the material. If material is not included in the article's Creative Commons licence and your intended use is not permitted by statutory regulation or exceeds the permitted use, you will need to obtain permission directly from the copyright holder. To view a copy of this licence, visit http://creativecommons.org/licenses/by/4.0/. The Creative Commons Public Domain Dedication waiver (http://creativecommons.org/publicdomain/zero/1.0/) applies to the data made available in this article, unless otherwise stated in a credit line to the data. 
EMT and immune cell infiltration. The most important finding was that these three genes were independent prognostic factors for BLCA.

Keywords: Wnt pathway, EMT, Computational biology, PPP2CB, Risk signature

\section{Introduction}

Bladder cancer (BLCA) is the tenth most usual cancer and ranks sixth among males. It is associated with high incidence and mortality rates. There are approximately 549,393 new cases and 199,922 deaths in the world annually [1]. The Wnt signaling pathway is a relatively classic signaling pathway related to cell differentiation, migration and cell polarity. The Wnt signaling pathway is fallen into three sub-pathways: the classic $\beta$-catenin-dependent path, the non-canonical Wnt/calcium path and the non-canonical planar cell polarity path. Abnormal Wnt signaling is related to some cancers, the most remarkable ones being colorectal, breast, lung, breast, oral, and cervical cancer and hematopoietic malignancies [2]. Most studies of the Wnt signaling pathway in BLCA only focused on the Wnt pathway as a downstream signaling pathway under the regulation of upstream molecules or medications to regulate the proliferation and differentiation of BLCA cells. These studies did not mention the influence of the Wnt signaling pathway on BLCA outcomes. Therefore, the current research determined the relationship between the Wnt signaling pathway and BLCA outcome and identified independent prognostic factors and the effects of Wnt pathway-related genes on BLCA outcome by using RNA-seq data downloaded from The Cancer Genome Atlas (TCGA). In addition, a prognostic model was set up on basis of recognized genes, the applicability and value of the model in BLCA were assessed; the generalizability of the model was studied using external verification using the Gene Expression Omnibus (GEO).

\section{Materials and approaches}

\section{Achievement of BLCA expression profiles from TCGA datasets}

UCSC Xena provided RNA-seq data (level 3) and clinical data of BLCA samples. The Genome Tissue Expression (GTEx) [3] tool was adopted to collect expression levels of genes discussed in normal tissues. Then, the "sva" package of R software [4] was used to normalize RNA expression profiles and remove batch effects. GSE13507 [5] was also obtained from GEO, the platform for which was GPL6102. The GSE13507 cohort contained 165 primary BLCA samples, 58 normal-looking bladder mucosa samples surrounding cancer, 23 recurrent non-muscle invasive tumor tissues, and ten normal bladder mucosae.
Analysis of clinical phenotype of the Wnt signaling path We downloaded the complete list of Wnt path genes from KEGG [6]. The list included several Wnt pathwayrelated genes (Additional file 1: Table S1). To identify the Wnt signaling pathway genes associated with BLCA outcome, we downloaded the relevant genes of the Wnt signaling pathway and performed univariate Cox regression analysis applying TCGA $(\mathrm{P}<0.05)$. To characterize Wnt-related gene expression patterns in BLCA, RNA sequencing data in TCGA samples were analyzed. Subsequently, based on the median value of Wnt pathway activation in the sample, all patients were fallen into two groups (activated and inhibited), and KEGG was used for difference analysis to create a volcano map.

\section{SsGSEA and WGCNA analysis for the analysis of Wnt-related biological functions}

Based on TCGA BLAD samples, single-sample gene set enrichment analysis (ssGSEA) [7] analysis was employed for the evaluation of 6935 Gene Oncology (GO) [8] biological functions of each sample. Five Wnt pathways were recognized applying weight gene co-expression network analysis (WGCNA) [9] clinical phenotype, and then module assignment analysis was performed based on WGCNA. We obtained the correlation diagrams for the ssGSEA scores of GO functions and the Wnt pathway. We found epithelial-mesenchymal transition (EMT)related biological functions were the GO terms most associated with the Wnt pathway. Use WGCNA (version: 1.61) for co-expression network analysis. First, we select the soft threshold for building the network. The soft threshold gives the adjacency matrix a continuous value between 0 and 1 , so that the established network meets the power law distribution and is near the actual state of the biological network. Second, we use the blockwise modules function to build a ladderless network, and then identify the gene co-expression modules that cluster genes with similar expression patterns by performing modulus assignment analysis. The definition of module is made by cutting the clustering tree into branches and assigning them different colors with the dynamic tree cutting algorithm. Then the Eigengene modulus (ME) of every modulus is calculated. ME represents the expression level of each module. Then, the association between the ME and the clinical characteristics of every module is calculated. At the end, the importance of genes in the 
computation module represents the association between genes and samples. The soft threshold for network construction was 5. Finally, we conducted in-depth research on the correlation between EMT and Wnt pathway genes.

\section{Consensus clustering and principal component analysis} Using the $\mathrm{R}$ software package "ConsensusClusterPlus" [10] from TCGA, based on the difference in expressing Wnt signaling pathway-associated genes in BLCA patients, we identified two subtypes (50 iterations, 80\% resampling rate Pearson correlation, http://www.bioco nductor.org/). We used the cumulative distribution function and consensus matrix to calculate the appropriate number of subtypes. Then, we drew Kaplan-Meier overall survival curves and the classification of clinical stages based on these two subtypes.

\section{Establishment and verification of risk scoring signature and line graph model}

The LASSO-Cox algorithm [11] in the "glmnet" R software package was employed to identify the overall survival clinical outcome signature. We verified the prognostic prediction value of the signature conducted in TCGA-BLAD by using Sangerbox (http://sangerbox. com/). Sangerbox is an R-based comprehensive analysis tool for bioinformatics.

\section{Analysis of three essential genes in the immune microenvironment}

As an algorithm, CIBERSORT [12] analyzes the ratio of cells in the body tissue gene expression matrix. As a genetic signature matrix, LM22 defines 22 immune cell subtypes that can be downloaded from the CIBERSORT website portal (https://cibersort.stanford.edu/). We used LM22 Matrix and cipher algorithm to analyze the ratio of $\mathrm{CD} 8+\mathrm{t}$ cells. The result showed that the number of samples was $\mathrm{P}<0.05$. Subsequently, the "ESTIMATE" $\mathrm{R}$ software package [13] was employed to measure tumor unit (referring to the ratio of cancer cells in tumor), immune mark (referring to the infiltration of immune cells in tumor) and matrix mark (recording the presence of tumor cells).We evaluated the immune microenvironment panorama of the factors in signatures to calculate correlations among the Wnt pathway, the immune microenvironment, and immune responses.

\section{Cell source and transfection}

The Cell Bank of the Chinese Academy of Sciences (China) provided BLCA cell lines (T24 and UM-UC-3). Both cell lines were grown at 37 degrees Celsius and 5\% $\mathrm{CO} 2$. Among them, the culture of T24 cells was made in RPMI (Hyclone; GE Healthcare), and the culture of UM-UC3 cells was made in high glucose DMEM (Hyclone; GE Healthcare). Both media were supplemented with $10 \%$ fetal bovine serum (FBS; Biological Industries, Beit-HaEmek, Israel). We ordered small interfering RNA (siRNA) for PPP2CB from JTSBIO Co. (China). The sequence is below si-PPP2CB (sense: GGA ACCAGGCUGCUAUCAUTT; anti-sense: AUGAUA GCAGCCUGGUUCCTT).

\section{Cell proliferation assay}

We use BeyoClick ${ }^{\mathrm{TM}}$ EdU-488 Cell Proliferation Kit (Beyotime Biotechnology, China) to measure cell proliferation. We incubated the transfected cells according to the instructions and obtained images on a fluorescence microscope (Olympus Corporation, Japan), and used ImageJ software (National Institutes of Health, Bethesda, Maryland) Perform a cell count.

\section{Wound healing assay}

We seeded the cells in a 6-well plate. After $48 \mathrm{~h}$ of transfection, when the cells reached confluence, we used a pipette tip to cut the cells to form a mechanical wound. Use an optical microscope to image at 0 and $24 \mathrm{~h}$, and compare cell migration by calculating the size of the gap in each field of view.

\section{Cell migration assay}

We put the $8 \mu \mathrm{m}$ Transwell chamber into a 24-well plate (Corning Costar, Corning, New York, USA), and preliminarily added $600 \mu \mathrm{l}$ of medium containing $10 \%$-FBS to each bottom well, and inoculated each upper chamber at the same time 8000 cells in suspension in $200 \mu \mathrm{l}$ FBS-free medium. After incubation at $5 \% \mathrm{CO} 2$ and $37{ }^{\circ} \mathrm{C}$ for $24 \mathrm{~h}$, the upper layer film was removed in the cells, while the cells migrated to the lower side is fixed in $4 \%$ paraformaldehyde, and stained with $1.0 \%$ crystal violet. Use a microscope to obtain an image.

\section{Immunoblotting assay}

We extract total protein from the cells by RIPA lysis buffer with 1\% PMSF, and then determine the protein concentration by the bicinchoninic acid assay (Beyotime Institute of Biotechnology). The protein samples isolated by PAGE are transferred to polyvinylidene fluoride membranes. Separate the membrane with anti-N-cadherin (1:5000, ab76011, Abcam, USA), antivimentin $(1: 1000,5741 \mathrm{~S}$, CST, USA), anti- $\beta$-tubulin (1:1000, 2128S, CST, USA) and anti-PРP2CB (1:10000; ab168371; Abcam, USA) antibodies were incubated overnight at $4{ }^{\circ} \mathrm{C}$. After that, the membrane and the secondary antibody were combined in a shaker at $37{ }^{\circ} \mathrm{C}$ for $1 \mathrm{~h}$, and the image was captured applying the EasySee Western Blot kit (Beijing Transgenic Biotechnology 
Co., Ltd., Beijing, China) and the chemiluminescence system (Bio-Rad), California, USA).

\section{Statistics analysis}

We applied the $\mathrm{R}$ packages "glmnet" and "survival" to perform multivariate, Kaplan-Meier analysis and LASSO-Cox regression to evaluate risk signature, and use the $\mathrm{R}$ package "survivalROC" to perform Roc curve analysis [14]. R software (version4.0.3; https:// www.r-project.org/) was used to perform all statistical analyses. It was considered that $\mathrm{P}<0.05$ was greatly significant. GraphPad Prism was also applied for data analysis.

\section{Results}

The relationship between Wnt signaling pathway-related genes and phenotypic characteristics of BLCA

On basis of the univariate Cox regression model, we found that 34 factors in the Wnt pathway were greatly related to OS. A forest map of risk scores displayed that 32 genes were risk elements and two genes (MAPK10 and WNT7B) were protective factors (Fig. 1A). The t-test of completely randomized data displayed the variation in the expression levels of these 34 genes in the clinical stages of BLCA and World Health Organization stages II and III (Fig. 1B). We used KEGG to analyze the metabolic pathways and signal transduction pathways of tumors significantly enriched in the Wnt pathway activation and

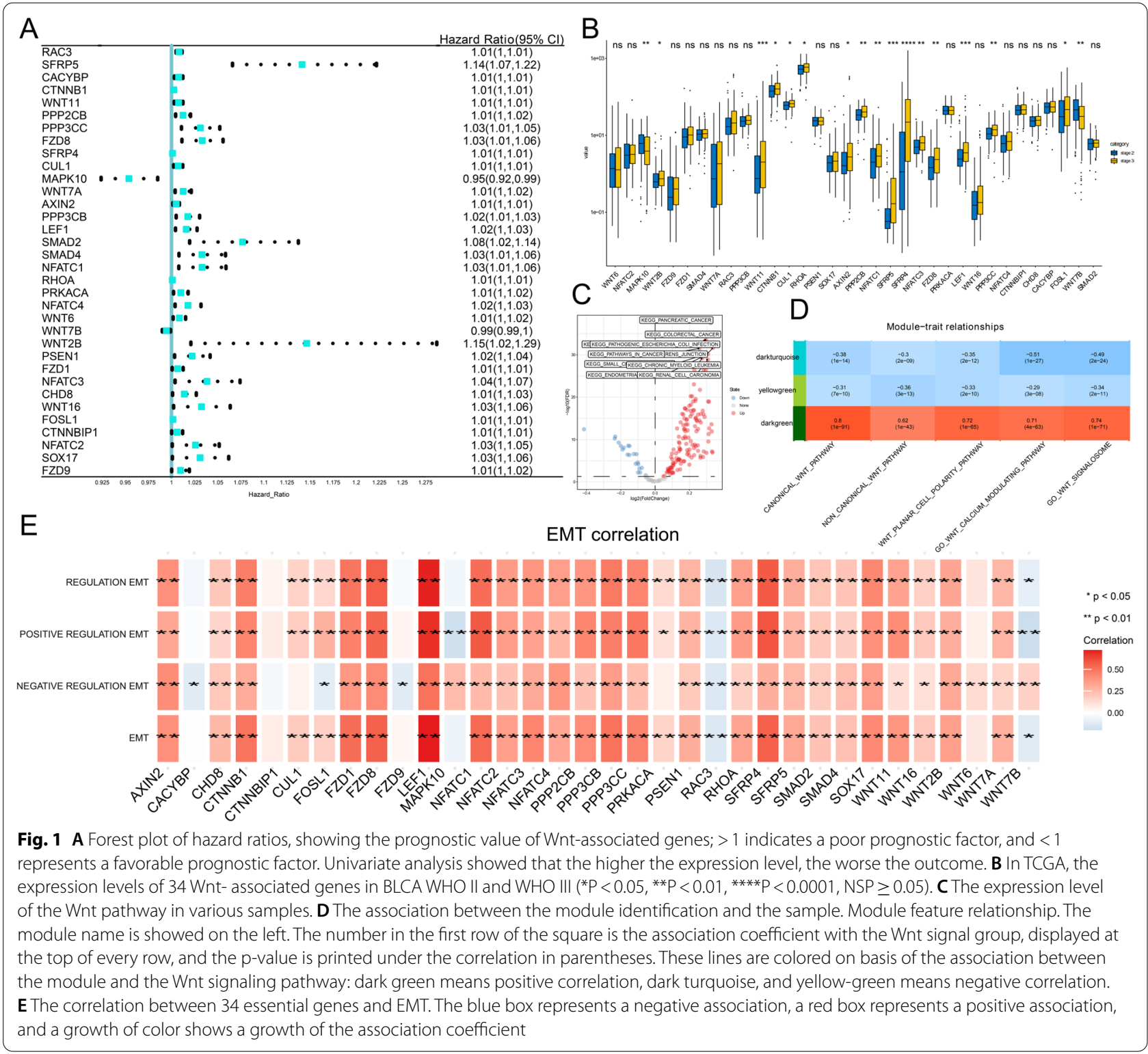


inhibition groups. We found that these tumor-related pathways were also highly activated (Fig. 1C). Therefore, we believe that the Wnt signal pathway promotes cancer.

\section{The association between the Wnt signaling pathway and biological functions in BLCA}

We used ssGSEA and WGCNA analysis to enrich the biological functions associated with the Wnt signaling pathway and tumors in TCGA. Three modules were obtained (dark turquoise, yellow-green and dark green) (Fig. 1D). Dark turquoise and yellow-green negatively correlated with Wnt pathways, and dark green positively correlated with Wnt pathways. In these three modules, we found that EMT-related biological GO terms were associated with the Wnt pathway (Table 1). EMT positively correlated with the activation of the Wnt signaling pathway. Therefore, we speculated that the high activation of Wnt signaling in BLCA would induce EMT in tumor cells, increasing tumor migration and invasion and affecting the outcome in terms of computational biology. Other biological functions were closely correlated with the activation or inhibition of Wnt signaling pathways, including angiogenesis, vascular endothelial cell migration, $G$ protein-coupled receptor pathways, and the mitochondrial respiratory chain (Table 1).

Table 1 Correlation among WNT pathway and other GO functions

\begin{tabular}{|c|c|c|c|c|c|}
\hline Correlation & $\begin{array}{l}\text { GO_CANONICAL_- } \\
\text { WNT_SIGNALING_- } \\
\text { PATHWAY }\end{array}$ & $\begin{array}{l}\text { GO_NON__ } \\
\text { CANONICAL_WNT__ } \\
\text { SIGNALING_PATHWAY }\end{array}$ & $\begin{array}{l}\text { GO_REGULATION__ } \\
\text { OF_WNT_SIGNALING_ } \\
\text { PATHWAY_PLANAR_ } \\
\text { CELL_POLARITY_ } \\
\text { PATHWAY }\end{array}$ & $\begin{array}{l}\text { GO_WNT_SIGNALING_ } \\
\text { PATHWAY_CALCIUM_ } \\
\text { MODULATING_ } \\
\text { PATHWAY }\end{array}$ & $\begin{array}{l}\text { GO_WNT_- } \\
\text { SIGNALOSOME }\end{array}$ \\
\hline $\begin{array}{l}\text { GO_EPITHELIAL_TO_ } \\
\text { MESENCHYMAL_TRANSI- } \\
\text { TION }\end{array}$ & 0.79480331 & 0.583747 & 0.677845813 & 0.749249036 & 0.762904048 \\
\hline $\begin{array}{l}\text { GO_G_PROTEIN_COU- } \\
\text { PLED_ACETYLCHO- } \\
\text { LINE_RECEPTOR_SIGN- } \\
\text { ALING_PATHWAY }\end{array}$ & 0.442942289 & 0.412743729 & 0.375230298 & 0.404088981 & 0.445448447 \\
\hline $\begin{array}{l}\text { GO_NEGATIVE_REGU- } \\
\text { LATION_OF_SPROUT- } \\
\text { ING_ANGIOGENESIS }\end{array}$ & 0.444334924 & 0.300323183 & 0.505019482 & 0.43604778 & 0.419748416 \\
\hline $\begin{array}{l}\text { GO_NEGATIVE_REGULA- } \\
\text { TION_OF_BLOOD_VES- } \\
\text { SEL_ENDOTHELIAL_- } \\
\text { CELL_MIGRATION }\end{array}$ & 0.443234707 & 0.310783794 & 0.462866401 & 0.427546524 & 0.406001389 \\
\hline $\begin{array}{l}\text { GO_G_PROTEIN_COU- } \\
\text { PLED_RECEPTOR_SIGN- } \\
\text { ALING_PATHWAY_COU- } \\
\text { PLED_TO_CYCLIC_- } \\
\text { NUCLEOTIDE_SECOND_- } \\
\text { MESSENGER }\end{array}$ & 0.631278967 & 0.545572181 & 0.703316308 & 0.522399396 & 0.540023623 \\
\hline GO_VASCULOGENESIS & 0.650854042 & 0.426591528 & 0.555919121 & 0.637577912 & 0.644784758 \\
\hline $\begin{array}{l}\text { GO_ACTIN_FILAMENT_ } \\
\text { BINDING }\end{array}$ & 0.734403861 & 0.686136572 & 0.692503374 & 0.590242774 & 0.624590603 \\
\hline $\begin{array}{l}\text { GO_MITOCHONDRIAL__ } \\
\text { RESPIRATORY_CHAIN__ } \\
\text { COMPLEX_IIIASSEMBLY }\end{array}$ & -0.589167212 & -0.52442356 & -0.482988077 & -0.547371294 & -0.589942517 \\
\hline $\begin{array}{l}\text { GO_RESPIRATORY_ELEC- } \\
\text { TRON_TRANSPORT_- } \\
\text { CHAIN }\end{array}$ & -0.50603574 & -0.470990736 & -0.403627026 & -0.556423377 & -0.540776029 \\
\hline $\begin{array}{l}\text { GO_RESPIRATORY_- } \\
\text { CHAIN_COMPLEX_III }\end{array}$ & -0.44836815 & -0.501114083 & -0.427534625 & -0.44733523 & -0.428247616 \\
\hline $\begin{array}{l}\text { GO_MITOCHONDRIAL_ } \\
\text { ELECTRON_TRANS- } \\
\text { PORT_UBIQUINOL_TO_ } \\
\text { CYTOCHROME_C }\end{array}$ & -0.425364356 & -0.46843121 & -0.418905826 & -0.441280101 & -0.403991375 \\
\hline $\begin{array}{l}\text { GO_STRUCTURAL_CON- } \\
\text { STITUENT_OF_RIBO- } \\
\text { SOME }\end{array}$ & -0.432828749 & -0.40109856 & -0.334483352 & -0.452632376 & -0.491683488 \\
\hline
\end{tabular}


According to these outcomes, the Wnt signaling pathway is closely related to EMT. We further explored the correlation between $34 \mathrm{Wnt}$ pathway-related genes that affect BLCA outcome and EMT. We calculated the influence of these 34 genes on EMT (Fig. 1E) and found that PPP2CB, LEF1, CTNNB1 were positively correlated with EMT. RAC3 was significantly negatively related to EMT.

\section{The subgroup of Wnt signal expression in BLCA}

Figure 1 illustrates univariate Cox analysis of 34 genes in the Wnt pathway that had significant regression coefficients and were associated with BLCA outcome and EMT. Based on these 34 genes, we classified the Wnt signaling pathway in patients with BLCA. The consensus clustering of Wnt-associated genes divides BLCA specimens into two clusters. Using the similarity with Wnt-related gene expression, we chose the value of $\mathrm{k}=2$ (Fig. 2A).

On basis of the expression of these 34 genes, the BLCA samples from TCGA data set were fallen into two subgroups (C1 and $\mathrm{C} 2)$. We compared the expression differences of these genes in these subgroups (Fig. 2B).
The high expression group was the $\mathrm{C} 1$ group, and the low expression group was the $\mathrm{C} 2$ group. The $\mathrm{C} 1$ group was the Wnt signaling pathway enrichment group, and the $\mathrm{C} 2$ group was the Wnt signaling pathway deficiency group. The $\mathrm{C} 1$ group had poor outcomes, and the $\mathrm{C} 2$ group had better outcomes. The risk ratio of the $\mathrm{C} 1$ group was 1.80 of the $\mathrm{C} 2$ group, and the confidence interval was 1.06-3.05 ( $\mathrm{P}=0.028)$ (Fig. 2C). According to the database, the proportions of clinical stages in the two groups differed. The proportion of stage II BLCA (WHO) in group $\mathrm{C} 1$ was $28 \%$; the proportion of stage II BLCA (WHO) in group C2 was $42 \%$; the proportion of stage IV BLCA (WHO) in group C1 was $36 \%$, and the proportion of BLCA in group C2 Phase III (WHO) accounted for $24 \%$. The difference between the two groups of Phase III (WHO) was small (Fig. 2D). These findings suggest that the BLCA outcome in Wnt signaling pathway enrichment group is worse. The outcome of stage IV patients was worse, the outcome of stage II patients was better, and no great variation was found between stage III patients and the Wnt signaling deficiency group.

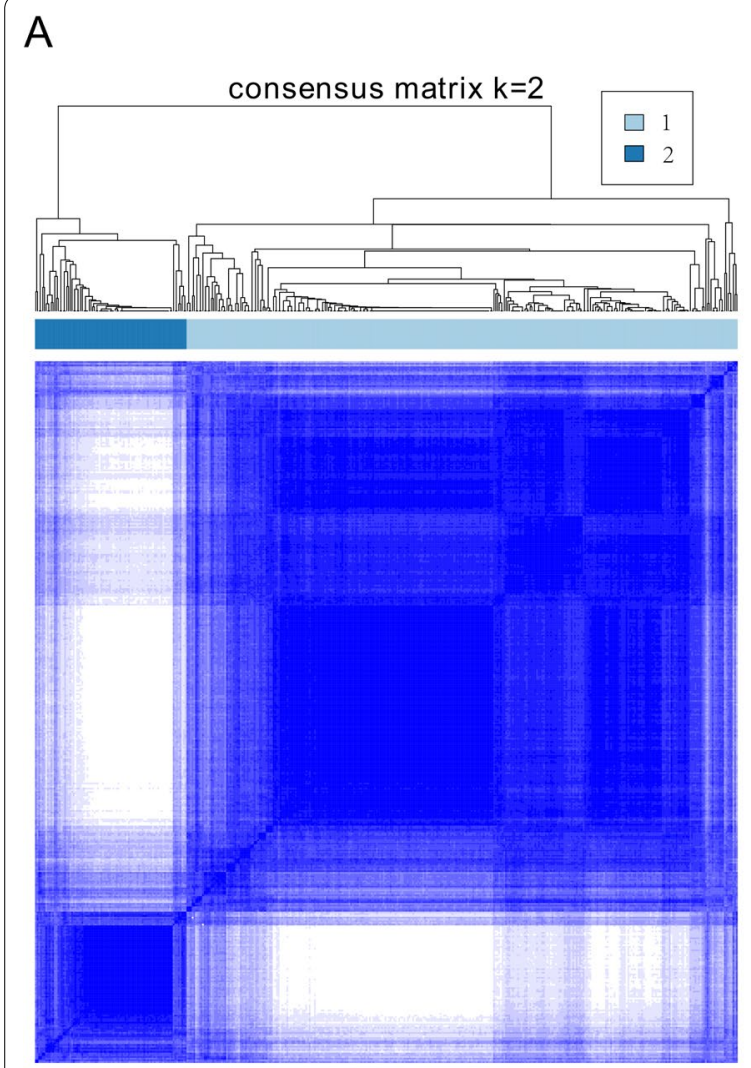

B
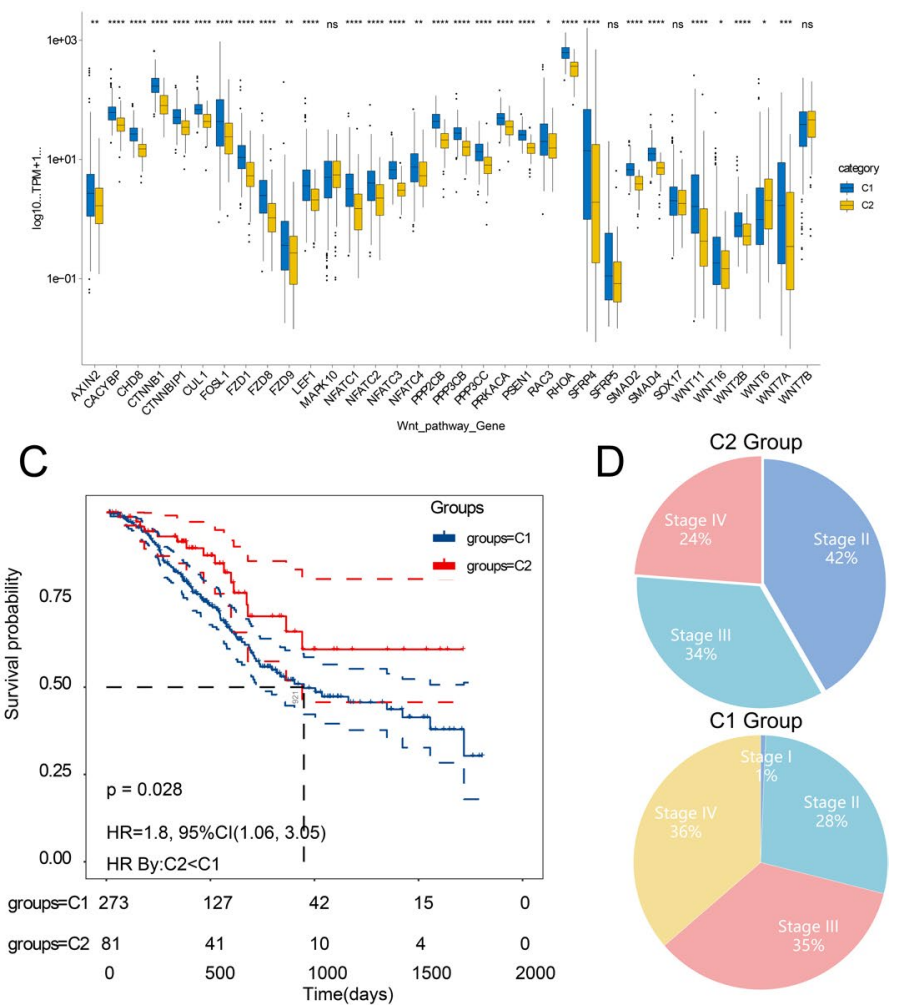

Fig. 2 A The most suitable $(k=2)$ consensus clustering matrix. B Differences in the expression of 34 Wnt signaling pathway-associated genes in the two subtypes of BLCA in TCGA data set. C Kaplan-Meier overall survival curves of BLCA patients with two subtypes in TCGA data set. Significance: ${ }^{*} P<0.05,{ }^{* *} P<0.01,{ }^{* *} P<0.001$. D Clinical staging of two 


\section{The prognostic prediction model associated with the Wnt} pathway in BLCA

Having found that the Wnt signaling pathway was related to BLCA outcome, we tried to establish a set of prognostic risk scores associated with the Wnt pathway based on BLCA. We found 34 Wnt pathway-related genes. After applying the LASSO-Cox algorithm to these 34 genes, we identified MAPK10, RAC3, and PPP2CB as independent prognostic genes (Fig. 3A, B). Considering the role of these three genes in BLCA outcome, a risk mark signature was set up for the integration of the effects of these genes using LASSO-Cox regression coefficients (Fig. 3C). Risk score $=-0.001 *$ MAPK10+0.002 $*$ RAC3 $+0.001 *$ PPP2CB.

Based on the median risk mark, BLCA patients were fallen into high-risk and low-risk groups in TCGA. The survival rate in the low-risk group was greatly higher than that of the high-risk group. According to the time-varying ROC curve, the region under the nomogram curve (AUC) used for the prediction of the 3-year survival rate was 0.62 , while the 5 -year survival rate predicted by the AUC was 0.6 (Fig. 3D).

Universality is an essential indicator for evaluating prognostic models, and it is necessary to verify signatures externally using data from other sources. GEO data sets applying various chip platforms were selected for the confirmation of the prognostic predictive power of Wntassociated genes and the signature. We found that these gene signatures had prognostic significance in GSE13507 (Fig. 4A-C).

The association between the three genes and the immune microenvironment of BLCA

Various immune infiltration and anti-tumor effects in the immune tumor microenvironment are caused by immune cells and immune-related pathways. The immune infiltration status of the transcriptome of BLCA data was assessed by CIBERSORT algorithm. Eight immunerelated genes in 22 immune cells were used to assess immune infiltration in BLCA. We analyzed the expression of MAPK10, RAC3, and PPP2CB in BLCA, the risk score, and the infiltration of immune cells (Fig. 5). By comparing with the MAPK10 high expression group, the MAPK10 low expression group had more M2 macrophages. Compared with the $\mathrm{PP} 2 \mathrm{CB}$ high expression group, the PPP2CB low expression group had fewer M2 macrophages (Fig. 5A).

The ESTIMATE algorithm was applied to assess the association between immune infiltration status and tumor purity, and the calculation of tumor stage, survival status, tumor purity, ESTIMATE mark, stroma mark, and the immune mark of TCGA was made (Fig. 5B). The heat map displayed that when the expression of MAPK10 is low, the purity of the tumor is lower, the immune score is higher, the stromal cell score is higher, and the expression of eight immune-associated genes is higher. When the expression of PPP2CB is low, the expression of the eight immune-related genes is low.

\section{Functional characteristics of PPP2CB}

To further validate our bioinformatics analysis, we selected a risk gene (PPP2CB) and performed cell function experiments by silencing its expression using siRNA. To date, no studies have reported the function of this gene in BLCA cells. We performed functional verification to determine the possible effects of this gene on the BLCA cell lines T24 and UM-UC3. After downregulating the expression of $\mathrm{PP} 2 \mathrm{CB}$, the proliferation and migration of BLCA cells were significantly inhibited (Figs. 6A, B; 7A). Using western blotting, it was observed that silencing PPP2CB led to downregulation of protein levels of $\mathrm{N}$-cadherin and vimentin, suggesting that this gene promotes EMT in BLCA cells (Fig. 7B). These results provide us with a part of the experimental basis for verifying bioinformatics analysis and provide further insights for the further study of molecular functions and mechanisms.

\section{Discussion}

Several biological phenomena were controlled by the Wnt signal transduction cascade throughout all animals' growth and adult life, and aberrant Wnt signaling emphasizes various pathologies in humans [15]. In this study, we found that Wnt signaling is related to the occurrence, growth, metastasis, and recurrence of BLCA, and the Wnt signaling pathway is a prognostic risk factor for BLCA.

In BLCA, many upstream and downstream molecular members of Wnt signaling participate in various biological functions. For example, Wnt signaling regulate

(See figure on next page.)

Fig. 3 A, B LASSO-Cox regression shows independent prognostic genes. The upper picture displays the shrinkage of the coefficients, and the lower picture shows a tenfold cross-validation. C Risk mark signature on basis of three essential genes. The upper figure displays the calculation formula and the value of the risk mark; the middle figure displays the distribution of survival status on basis of the risk mark; the bottom figure displays the cluster heat map of the three essential genes. D ROC over time shows the accuracy of Nomogram in predicting 3-year overall survival and 5-year overall survival. According to the Kaplan-Meyer survival curve, the survival rates of BLCA patients with high-risk scores and low-risk scores are different 
A

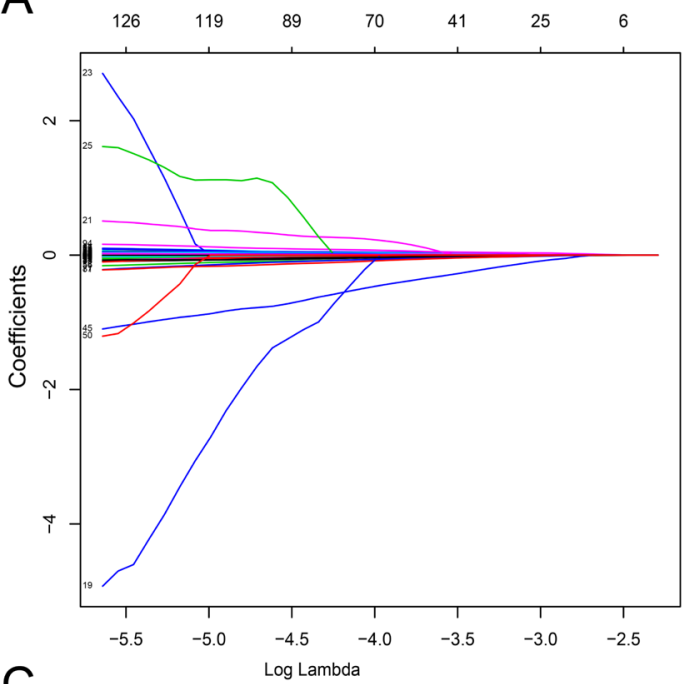

B

$\begin{array}{llllllllllllll}127 & 124 & 119 & 104 & 89 & 78 & 64 & 48 & 35 & 28 & 14 & 7 & 3 & 0\end{array}$

C
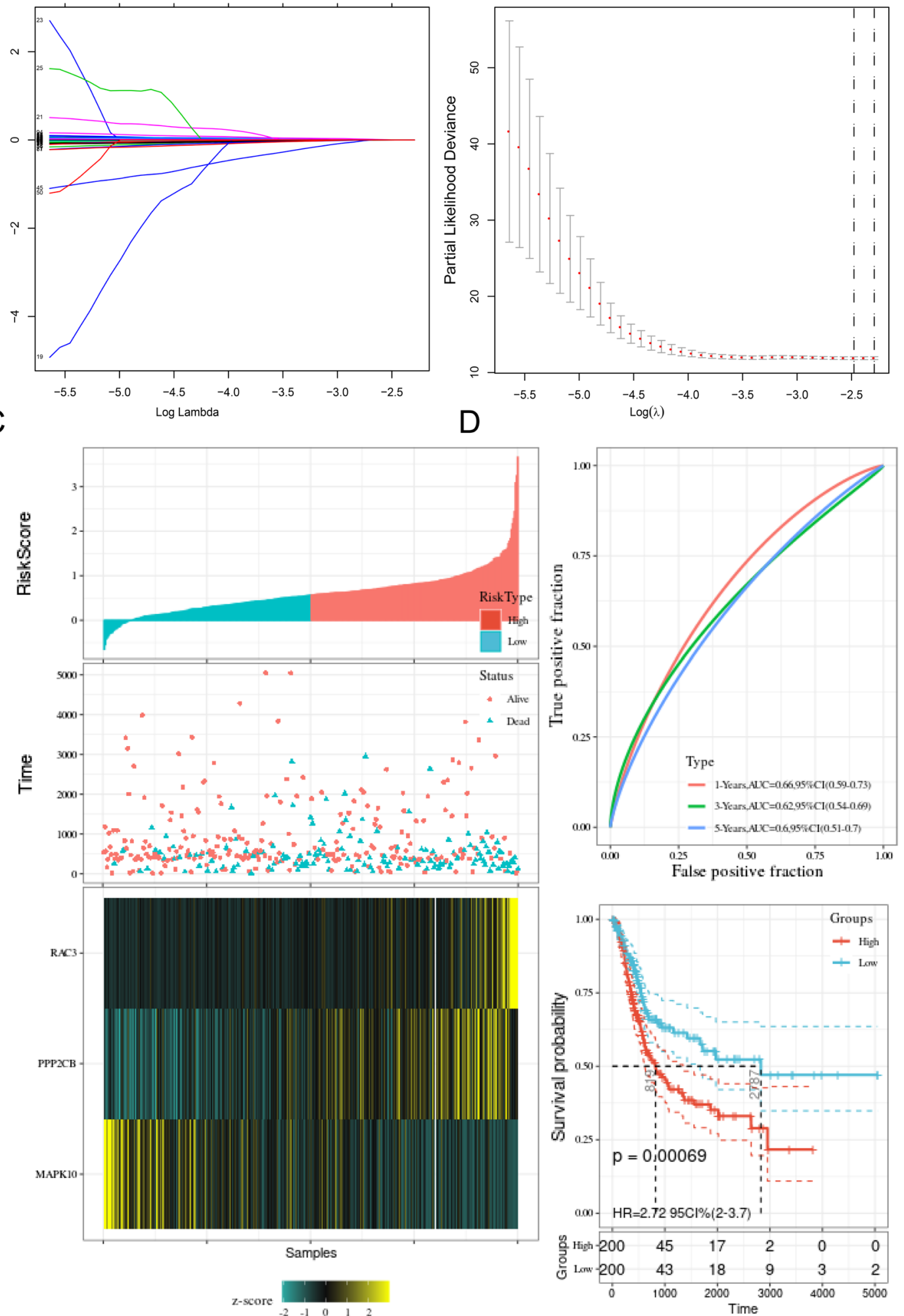

Fig. 3 (See legend on previous page.) 

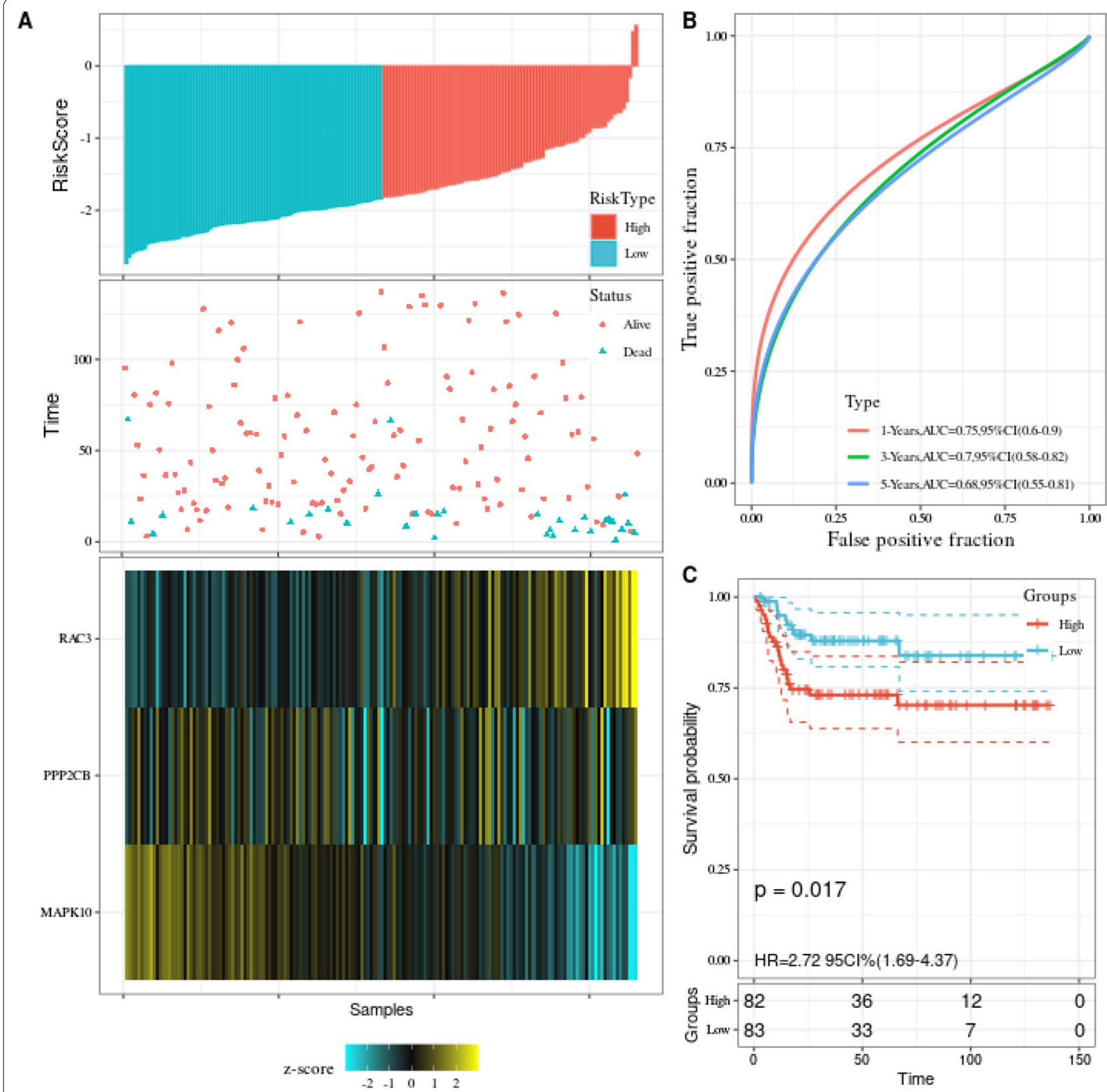

Fig. 4 Confirm the prognostic value of the three Wnt-related genes in the GEO dataset. A Risk mark signature on basis of three basic genes. The upper figure displays the calculation formula and the value of the risk mark; the middle figure displays the distribution of survival status on basis of the risk mark; the bottom figure displays the cluster heat map of the three essential genes. B Time-dependent ROC displays the accuracy of the nomogram of 1-year overall survival prediction, 3-year overall survival prediction, and 5-year overall survival prediction. C Kaplan-Meyer survival curve displays the survival rate of BLCA patients with high-risk and low-risk marks

angiogenesis [16-19], induces vascular endothelial cell migration [20, 21], and participates in G-protein-coupled receptor signal transmission [22-24], regulation of mitochondrial respiratory chain [25-28], and EMT.

Based on the WGCNA analysis, we found EMT-related GO terms strongly correlated with Wnt pathways. Therefore, we focused on the regulation mechanism of EMT and Wnt. EMT is a necessary process in morphogenesis. Epithelial cells lose cell-to-cell contact, polarity, and other epithelial cells' characteristics and obtain the unique characteristics of mesenchymal cells (including enhanced motility). The characteristics of EMT are the loss of E-cadherin on the plasma membrane, the increase of vimentin and fibronectin, and the growing 


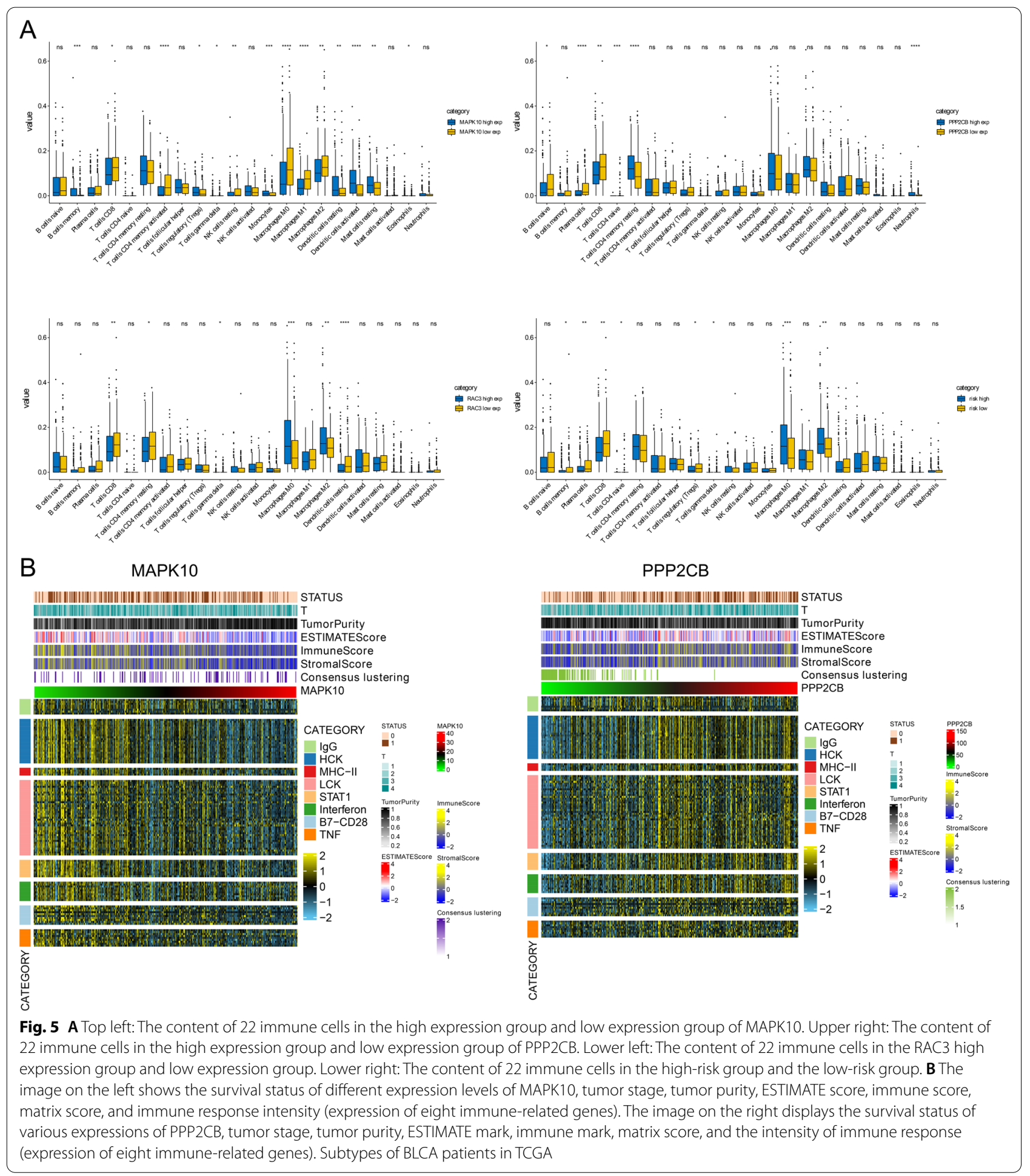

accumulation of $\beta$-catenin in the nucleus. Except normal growth processes, EMT also participates in tumor development and progression. The initiation of tumor metastasis depends on the interaction between tumor cells and stromal cells, and EMT occurs in single cells. Hybrid
EMT occurs in collectively migrating cells [29]. These findings suggest that EMT is closely related to tumor metastasis and recurrence.

EMT is regulated by signal transduction pathways including TGF- $\beta$, Notch, NF-kB, Wnt, and receptor 


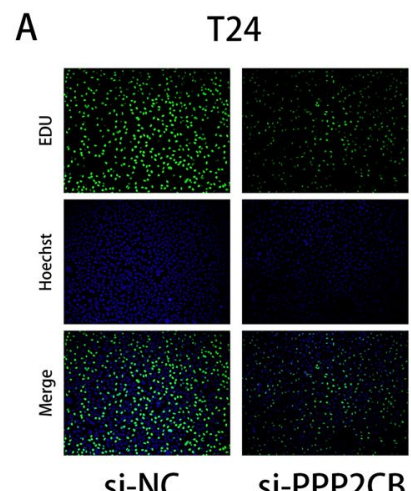

B

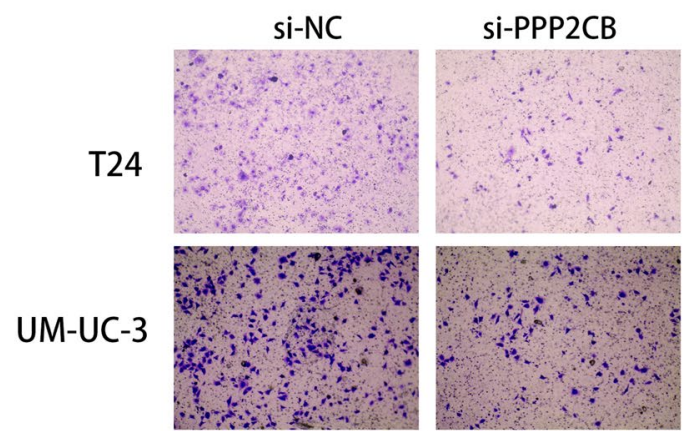

UM-UC-3

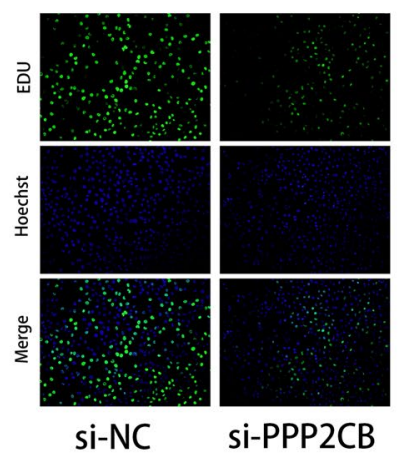

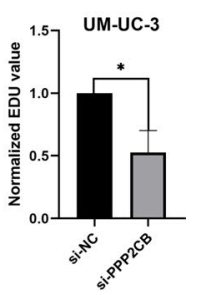
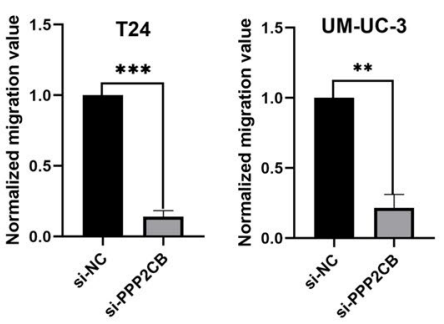

Fig. 6 A The proliferation of T24 and UM-UC-3 after silencing the expressions of PPP2CB. B Migration of T24 and UM-UC-3 after silencing the expressions of PPP2CB. The analysis of data was made using the t-test, which was showed as the mean \pm standard deviation

tyrosine kinase [30]. The regulation of EMT by the Wnt signaling pathway has been determined including Wnt5a [31], FOXP3 [32], SERPINH1 [33], CUL4B [34], and SUFU [35], which can be used in BLCA, gastric cancer, and pancreatic cancer. Activating the Wnt signaling pathway induces EMT. The Wnt pathway also inhibits EMT, and EFEMP2 may inhibit BLCA progression through the Wnt/ $\beta$-catenin pathway to prevent EMT [36]. The downregulation of E-cadherin causes the nuclear translocation of $\beta$-catenin and activation of canonical Wnt signaling [37]. As a marker gene of EMT, the gene slug also induces nuclear translocation of $\beta$-catenin [38]. Studies have shown a close association between the Wnt signaling pathway and EMT and two-way modulation between the two.

Because BLCA often recurs and has high invasiveness and metastasis, we explored the correlation between the Wnt signaling pathway and EMT and the impact of the Wnt pathway on BLCA outcome. We found that patients with highly activated Wnt signaling pathways had a more unsatisfactory outcome, suggesting that the Wnt signaling pathway is cancer-promoting. We also obtained five important prognostic independent risk factors for BLCA based on the Wnt pathway (MAPK10, PPP2CB, LEF1, CTNNB1, and RAC3). These Wnt signaling pathway genes are closely associated with EMT. PPP2CB, LEF1, CTNNB1 positively correlated with EMT; RAC3 significantly negatively correlated with EMT; MAPK10 positively regulated and inhibited EMT.

Wnt signal transduction and its crosstalk with various immune cells have both positive and negative effects on tumor progression. For example, it can renew white blood cells or it can promote immune tolerance and limit the anti-tumor response.

The interaction between inflammatory cells and cancer cells has been researched well. Signal pathways such as TGF- $\beta$, NF- $\mathrm{kB}$ and Notch have been confirmed to affect the occurrence of EMT. Changes in the tumor microenvironment are also closely related to tumor proliferation and metastasis, affecting the development of cancer and the prognosis of patients. Pro-inflammatory factors and immune-related factors also play different roles in the process of tumor metastasis. For example, mitochondrial reactive oxygen species (ROS) can further affect tumor metastasis by regulating the inflammatory response [3941]. Thus some natural antioxidants or anti-inflammatory drugs may also be used to inhibit tumor metastasis $[39,42,43]$. In colorectal cancer, including the spectrum from normal colorectal adenoma to cancer, infiltrating macrophages display high levels of Wnt2 and Wnt5a. 


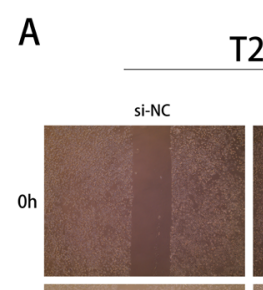

T24
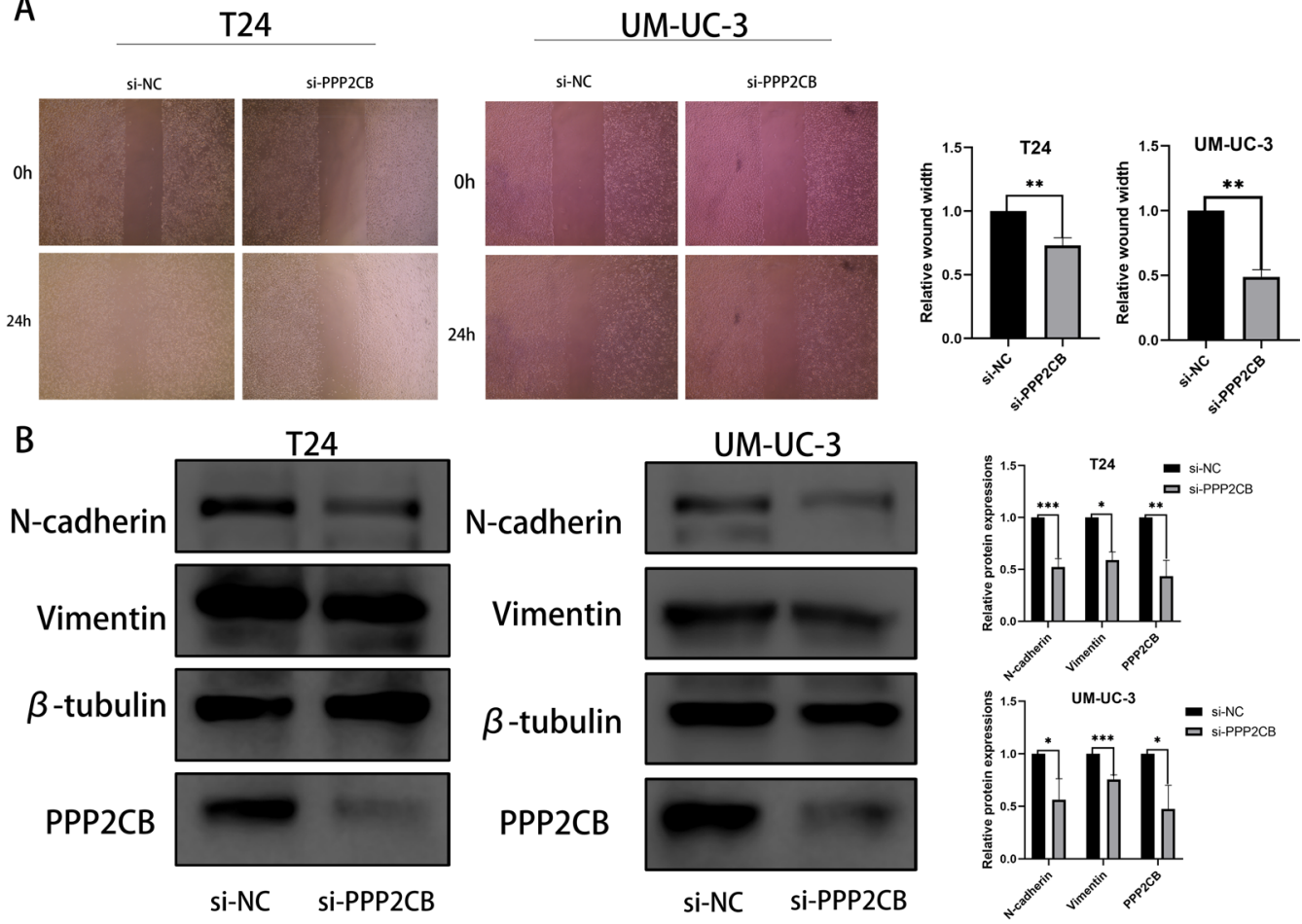

Fig. 7 A Migration of T24 and UM-UC-3 after silencing the expressions of PPP2CB. The analysis of data was made by t-test, which was showed as the mean \pm standard deviation. B The detection of PPP2CB, vimentin and $\mathrm{N}$-cadherin proteins in T24 and UM-UC-3 was made after silencing the expressions of PPP2CB. ${ }^{* P}<0.05 ;{ }^{* *} \mathrm{P}<0.01 ;{ }^{* *} \mathrm{P}<0.001$

This finding suggests that the activation of paracrine Wnt by macrophages leads to cancer progression [44]. The current research showed that the $\mathrm{Wnt} / \beta$-catenin pathway-related factors of the Wnt classic pathway andB cell transcription coactivator 9 (BCL9) were related to immune cell infiltration. The expression of BCL9/BCL9L negatively related to the infiltration of $\mathrm{CD} 8+\mathrm{T}$ cells in triple negative breast cancer, and BCL9/BCL9L inhibited the infiltration of $\mathrm{CD} 8+\mathrm{T}$ cells in the tumor microenvironment [45].

The Wnt pathway promotes tumor growth by inhibiting immune cell infiltration. Wnt ligands secreted by tumor cells stimulate tumor-related macrophages to polarize to the M2 subtype through the canonical Wnt signaling pathway, leading to tumor development and migration [46]. This finding was consistent with our results, showing that when PPP2CB is highly expressed, the infiltration of M2-type macrophages is more substantial. M2 type macrophages have lost the functions of tumor antigen peptide presentation and processing. When the number of M2 type macrophages increases, immunity weakens, and outcomes worsen, suggesting that $\mathrm{PPP} 2 \mathrm{CB}$ is an oncogene. When the expression of MAPK10 is low, the infiltration of M2-type macrophages is more substantial, suggesting that MAPK10 is a tumor suppressor gene.
In summary, as the central transportation hub in tumor tissue, PPP2CB affects tumor cell migration by regulating EMT, and it also regulates the tumor immune microenvironment and affects tumor immunity by interacting with immune cells.

\section{Conclusion}

We set up a three-gene signature on basis of Wnt signaling-related genes, EMT, and immune infiltration in BLCA. These genes affect tumor proliferation as members of the Wnt pathway family and affect tumor metastasis by regulating EMT, even participating in immune infiltration. We also identified PPP2CB as a risk factor for the activity, proliferation, and migration of BLCA cells and established relevant immune infiltration assessments to predict their survival rate accurately. We expect that our findings will deepen the understanding of the molecular mechanisms taking part in the occurrence and development of BLCA and provide a unique method for discovering predictive biomarkers and selecting targeted therapies.

\section{Abbreviations}

CDF: Cumulative distribution function; ME: Module Eigengene; GS: Gene significance; GTEx: Genome Tissue Expression; ROC: Receiver Operating 
Characteristic; BLCA: Bladder cancer; WGCNA: Weighted correlation network analysis; EMT: Epithelial-mesenchymal transition; RNA-seq: RNA-sequencing; TCGA: The Cancer Genome Atlas; GEO: Gene Expression Omnibus; NMF: NonNegative Matrix Factorization; ssGSEA: Single-sample gene set enrichment analysis; GSVA: Gene Set Variation Analysis; OS: Overall survival; BCL9: B cell transcription coactivator 9; ROS: Mitochondrial reactive oxygen species.

\section{Supplementary Information}

The online version contains supplementary material available at https://doi. org/10.1186/s12967-021-03061-4.

Additional file 1. Table S1. The list of WNT pathway genes name in KEGG.

\section{Acknowledgements}

We would like to thank TCGA and GEO for their free use.

\section{Authors' contributions}

SS, YW, and JB designed the study; SS and YW analyzed and wrote the manuscript. All authors have read and contented to the final version of the manuscript. SS and YW made equal contributions to this article. All authors read and approved the final manuscript.

\section{Funding}

China Shenyang Science and Technology Plan (20-205-4-015) supported this work.

\section{Declarations}

Ethics approval and consent to participate

Not applicable.

\section{Consent for publication}

Not applicable.

\section{Competing interests}

No competing interests were declared.

Received: 8 June 2021 Accepted: 30 August 2021

Published online: 27 September 2021

\section{References}

1. Bray F, Ferlay J, Soerjomataram I, Siegel RL, Torre LA, Jemal A. Global cancer statistics 2018: GLOBOCAN estimates of incidence and mortality worldwide for 36 cancers in 185 countries. CA Cancer J Clin. 2018;68(6):394-424

2. Patel S, Alam A, Pant R, Chattopadhyay S. Wnt signaling and its significance within the tumor microenvironment: novel therapeutic insights. Front Immunol. 2019;10:2872

3. Lonsdale J, Thomas J, Salvatore M, Phillips R, Lo E, Shad S, Hasz R, Walters G, Garcia F, Young N, Foster B. The genotype-tissue expression (GTEx) project. Nat Genet. 2013;45(6):580-5.

4. Leek JT, Johnson WE, Parker HS, Jaffe AE, Storey JD. The sva package for removing batch effects and other unwanted variation in high-throughput experiments. Bioinformatics. 2012;28(6):882-3.

5. Lee JS, Leem SH, Lee SY, Kim SC, Park ES, Kim SB, et al. Expression signature of E2F1 and its associated genes predict superficial to invasive progression of bladder tumors. J Clin Oncol. 2010;28(16):2660-7.

6. Kanehisa M, Goto S. KEGG: kyoto encyclopedia of genes and genomes. Nucleic Acids Res. 2000;28(1):27-30.

7. Subramanian A, Tamayo P, Mootha VK, Mukherjee S, Ebert BL, Gillette MA, et al. Gene set enrichment analysis: a knowledge-based approach for interpreting genome-wide expression profiles. Proc Natl Acad Sci U S A. 2005;102(43):15545-50.
8. Ashburner M, Ball CA, Blake JA, Botstein D, Butler H, Cherry JM, et al Gene ontology: tool for the unification of biology. Gene Ontol Consortium Nat Genet. 2000:25(1):25-9.

9. Langfelder P, Horvath S. WGCNA: an R package for weighted correlation network analysis. BMC Bioinform. 2008;9:559.

10. Wilkerson MD, Hayes DN. ConsensusClusterPlus: a class discovery tool with confidence assessments and item tracking. Bioinformatics. 2010;26(12):1572-3.

11. Tibshirani R. The lasso method for variable selection in the Cox model. Stat Med. 1997;16(4):385-95.

12. Newman AM, Liu CL, Green MR, Gentles AJ, Feng W, Xu Y, et al. Robust enumeration of cell subsets from tissue expression profiles. Nat Methods. 2015;12(5):453-7.

13. Yoshihara K, Shahmoradgoli M, Martínez E, Vegesna R, Kim H, TorresGarcia W, et al. Inferring tumour purity and stromal and immune cell admixture from expression data. Nat Commun. 2013;4:2612.

14. Heagerty PJ, Lumley T, Pepe MS. Time-dependent ROC curves for censored survival data and a diagnostic marker. Biometrics. 2000;56(2):337-44.

15. Clevers $H$, Nusse R. Wnt/ $\beta$-catenin signaling and disease. Cell. 2012;149(6):1192-205

16. Jiang $L$, Yin $M$, Wei $X$, et al. Bach1 represses Wnt/ $\beta$-catenin signaling and angiogenesis. Circ Res. 2015;117(4):364-75.

17. Vallée A, Guillevin R, Vallée JN. Vasculogenesis and angiogenesis initiation under normoxic conditions through Wnt/ $\beta$-catenin pathway in gliomas. Rev Neurosci. 2018;29(1):71-91.

18. Park H, Yamamoto H, Mohn L, et al. Integrin-linked kinase controls retinal angiogenesis and is linked to Wnt signaling and exudative vitreoretinopathy. Nat Commun. 2019;10(1):5243.

19. Shetti D, Zhang B, Fan C, Mo C, Lee BH, Wei K. Low dose of paclitaxel combined with XAV939 attenuates metastasis, angiogenesis and growth in breast cancer by suppressing Wnt signaling. Cells. 2019:8(8):892.

20. Vallée A, Lecarpentier Y. Crosstalk between peroxisome proliferatoractivated receptor gamma and the canonical WNT/ $\beta$-catenin pathway in chronic inflammation and oxidative stress during carcinogenesis. Front Immunol. 2018;9:745

21. Lyle CL, Belghasem M, Chitalia VC. c-Cbl: an important regulator and a target in angiogenesis and tumorigenesis. Cells. 2019;8(5):498.

22. Huch M, Dorrell C, Boj SF, et al. In vitro expansion of single Lgr5+ liver stem cells induced by Wnt-driven regeneration. Nature. 2013:494(7436):247-50.

23. Leung C, Tan SH, Barker N. Recent advances in Lgr5(+) stem cell research. Trends Cell Biol. 2018;28(5):380-91.

24. Wu Y, Chen W, Gong L, Ke C, Wang H, Cai Y. Elevated G-protein receptor 125 (GPR125) expression predicts good outcomes in colorectal cancer and inhibits Wnt/ $\beta$-catenin signaling pathway. Med Sci Monit. 2018;24:6608-16.

25. Godoy JA, Arrázola MS, Ordenes D, Silva-Alvarez C, Braidy N, Inestrosa NC. Wnt-5a ligand modulates mitochondrial fission-fusion in rat hippocampal neurons. J Biol Chem. 2014;289(52):36179-93.

26. Lee SY, Jeon HM, Ju MK, et al. Dlx-2 is implicated in TGF- $\beta$ - and Wntinduced epithelial-mesenchymal, glycolytic switch, and mitochondrial repression by Snail activation. Int J Oncol. 2015;46(4):1768-80.

27. Zhang W, Sviripa VM, Kril LM, et al. An underlying mechanism of dual Wnt inhibition and ampk activation: mitochondrial uncouplers masquerading as Wnt inhibitors. J Med Chem. 2019;62(24):11348-58.

28. Xia SR, Wen XY, Fan XL, et al. Wnt2 overexpression protects against PINK1 mutant-induced mitochondrial dysfunction and oxidative stress. Mol Med Rep. 2020;21(6):2633-41.

29. Majidpoor J, Mortezaee K. Steps in metastasis: an updated review. Med Oncol. 2021:38(1):3.

30. Jing Y, Han Z, Zhang S, Liu Y, Wei L. Epithelial-mesenchymal transition in tumor microenvironment. Cell Biosci. 2011:1:29.

31. Lopez-Bergami P, Barbero G. The emerging role of Wnt5a in the promotion of a pro-inflammatory and immunosuppressive tumor microenvironment. Cancer Metastasis Rev. 2020;39(3):933-52.

32. Yang S, Liu Y, Li MY, et al. FOXP3 promotes tumor growth and metastasis by activating Wnt/ $\beta$-catenin signaling pathway and EMT in nonsmall cell lung cancer. Mol Cancer. 2017;16(1):124. 
33. Tian $S$, Peng $P$, Li J, et al. SERPINH1 regulates EMT and gastric cancer metastasis via the $\mathrm{Wnt} / \beta$-catenin signaling pathway. Aging. 2020;12(4):3574-93.

34. He YM, Xiao YS, Wei L, Zhang JQ, Peng CH. CUL4B promotes metastasis and proliferation in pancreatic cancer cells by inducing epithelialmesenchymal transition via the Wnt/ $\beta$-catenin signaling pathway. J Cell Biochem. 2018;119(7):5308-23.

35. Peng $Y$, Zhang $X$, Lin $H$, et al. SUFU mediates EMT and Wnt/ $\beta$-catenin signaling pathway activation promoted by miRNA-324-5p in human gastric cancer. Cell Cycle. 2020;19(20):2720-33.

36. Zhou Q, Chen S, Lu M, et al. EFEMP2 suppresses epithelial-mesenchymal transition via $\mathrm{Wnt} / \beta$-catenin signaling pathway in human bladder cancer. Int J Biol Sci. 2019;15(10):2139-55.

37. Huels DJ, Ridgway RA, Radulescu S, et al. E-cadherin can limit the transforming properties of activating $\beta$-catenin mutations. EMBO J. 2015;34(18):2321-33.

38. Conacci-Sorrell M, Simcha I, Ben-Yedidia T, et al. Autoregulation of E-cadherin expression by cadherin-cadherin interactions: the roles of beta-catenin signaling, Slug, and MAPK. J Cell Biol. 2003;163(4):847-57.

39. Zhu Z, Jiang T, Suo H, Xu S, Zhang C, Ying G, et al. Metformin potentiates the effects of anlotinib in NSCLC via AMPK/mTOR and ROS-mediated signaling pathways. Front Pharmacol. 2021;12:712181.

40. Sawai H, Funahashi H, Okada Y, Matsuo Y, Sakamoto M, Yamamoto M, et al. Interleukin-1alpha enhances IL-8 secretion through p38 mitogenactivated protein kinase and reactive oxygen species signaling in human pancreatic cancer cells. Med Sci Monit. 2005;11(10):BR343-50.

41. Reynaert NL, van der Vliet A, Guala AS, McGovern T, Hristova M, Pantano C, et al. Dynamic redox control of NF-kappaB through glutaredoxin-regulated S-glutathionylation of inhibitory kappaB kinase beta. Proc Natl Acad Sci U S A. 2006;103(35):13086-91.

42. Nguyen TT, Ung TT, Li S, Lian S, Xia Y, Park SY, et al. Metformin inhibits lithocholic acid-induced interleukin 8 upregulation in colorectal cancer cells by suppressing ROS production and NF-kB activity. Sci Rep. 2019;9(1):2003.

43. Ahmadian E, Eftekhari A, Babaei H, Nayebi AM, Eghbal MA. Anti-cancer effects of citalopram on hepatocellular carcinoma cells occur via cytochrome $\mathrm{c}$ release and the activation of NF-kB. Anticancer Agents Med Chem. 2017;17(11):1570-7.

44. Smith K, Bui TD, Poulsom R, Kaklamanis L, Williams G, Harris AL. Upregulation of macrophage wnt gene expression in adenoma-carcinoma progression of human colorectal cancer. Br J Cancer. 1999;81(3):496-502.

45. Wang $X$, Feng $M$, Xiao T, et al. BCL9/BCL9L promotes tumorigenicity through immune-dependent and independent mechanisms in triple negative breast cancer. Oncogene. 2021;40(16):2982-97.

46. Yang Y, Ye YC, Chen Y, et al. Crosstalk between hepatic tumor cells and macrophages via Wnt/ $\beta$-catenin signaling promotes M2-like macrophage polarization and reinforces tumor malignant behaviors. Cell Death Dis. 2018;9(8):793.

\section{Publisher's Note}

Springer Nature remains neutral with regard to jurisdictional claims in published maps and institutional affiliations.
Ready to submit your research? Choose BMC and benefit from:

- fast, convenient online submission

- thorough peer review by experienced researchers in your field

- rapid publication on acceptance

- support for research data, including large and complex data types

- gold Open Access which fosters wider collaboration and increased citations

- maximum visibility for your research: over $100 \mathrm{M}$ website views per year

At BMC, research is always in progress.

Learn more biomedcentral.com/submissions 